\title{
BMJ Open Selective carious tissue removal using subjective criteria or polymer bur: study protocol for a randomised controlled trial (SelecCT)
}

\author{
Falk Schwendicke, ${ }^{1}$ Soraya Leal, ${ }^{2}$ Peter Schlattmann, ${ }^{3}$ Sebastian Paris, ${ }^{1}$ \\ Ana Paula Dias Ribeiro, ${ }^{4}$ Marta Gomes Marques, ${ }^{2}$ Leandro Augusto Hilgert ${ }^{2}$
}

To cite: Schwendicke F, Leal S, Schlattmann P, et al. Selective carious tissue removal using subjective criteria or polymer bur: study protocol for a randomised controlled trial (SelecCT). BMJ Open 2018;8:e022952. doi:10.1136/ bmjopen-2018-022952

- Prepublication history for this paper is available online. To view these files, please visit the journal online (http://dx.doi. org/10.1136/bmjopen-2018022952).

Received 15 March 2018 Revised 28 June 2018 Accepted 9 October 2018

Check for updates

(C) Author(s) (or their employer(s)) 2018. Re-use permitted under CC BY-NC. No commercial re-use. See rights and permissions. Published by BMJ.

${ }^{1}$ Department of Operative and Preventive Dentistry, CharitéUniversitätsmedizin, Berlin,

Germany

${ }^{2}$ Department of Dentistry, School of Health Sciences, University of Brasília, Brasilia, Brazil

${ }^{3}$ Department of Medical

Statistics, Informatics and

Documentation, Jena University

Hospital, Jena, Germany

${ }^{4}$ Department of Restorative Dental Sciences, University of Florida College of Dentistry, Gainesville, Florida, USA

Correspondence to

Dr Falk Schwendicke;

falk.schwendicke@charite.de

\section{ABSTRACT}

Introduction Selective (incomplete/partial) carious tissue removal is suitable for treating deep carious lesions in teeth with vital, asymptomatic pulps. In the periphery of a cavity, removal to hard dentin is performed, while in pulpo-proximal areas, leathery or soft dentin is left to avoid pulp exposure. As the decision of what contains 'soft' or 'leathery' dentin is subjective, using self-limiting burs which help to standardise the hardness of the remaining dentin, has been suggested to increase the reliability of carious tissue removal. The trial compares subjectively measured selective carious tissue removal in deep lesions in primary teeth with objectively measured selective removal with a self-limiting bur (Polybur, Komet).

Methods and analysis A community-based single-blind clustered randomised controlled superiority trial nested into a larger evaluation is performed. Recruitment for this trial has been concluded. We have recruited 115 children aged $6-8$ years with $\geq 1$ vital primary molar with a deep dentin lesion. The unit of randomisation was the child, with all eligible molars per child treated identically. Treatment was performed in a mobile dental unit. Subjective and objective carious tissue removal was performed at random. Teeth were restored using glass ionomer cement (Equia Forte, GC). Our primary outcome will be the time until complications occur, evaluated via multilevel survival analysis. Secondary outcomes will be the time until extraction is needed, subjective satisfaction of the child with the treatment (measured using a Likert scale) and cost-effectiveness. Re-examination will be performed after 12,24 and 36 months (the final examination is expected in 2020).

Ethics and dissemination This trial has been approved by the Ethics Committee of the Health Sciences of the University of Brasilia (CAAE 51310415.0.0000.0030). Trial results will be published in peer-reviewed journals and presented on conferences.

Trial registration number NCT02754466.

\section{INTRODUCTION}

Treating deep carious lesions in primary teeth with vital pulps and without pulpal symptoms is challenging: conventional 'complete' carious tissue removal aiming to remove all
Strengths and limitations of this study

The target population is a high-needs population.

- A randomised design will be used to compare different removal strategies.

- Blinding of the examiners will ensure low risk of detection bias.

- The absence of operator and patient blinding is a limitation of the study.

- A comprehensive set of clinical, subjective and health-economic outcomes will be used.

infected or affected dentin risks exposing the pulp. ${ }^{1}$ Pulpal exposure in primary teeth requires more invasive and complex therapies like pulpotomy or removal of the tooth, which are painful and can be traumatic. For children with limited compliance and high dental fear, such treatments can oftentimes only be provided under sedation or general anaesthetics, coming with the risks of systemic adverse events. Moreover, these treatments generate costs, and follow-up treatments are required (restoration or tooth removal in case of pulpotomy, orthodontic alignment or replacement of the tooth in case of extraction), which further impact on the child and the cost-effectiveness of the treatment. $^{2}$

Thus, avoiding pulp exposure is important when treating vital primary teeth with deep lesions. Selective carious tissue removal has been advocated for this purpose, as carious dentin is left in proximity to the pulp to reduce the risk of pulpal exposure, while removal peripherally aims to leave only hard dentin. The cavity is then sealed with a restoration; an intact seal is thought to deprive remaining bacteria from carbohydrates and thereby arrest the lesion. ${ }^{3}$ Numerous studies have demonstrated the clinical benefits of this approach, especially in primary teeth. ${ }^{4}$ 
However, doubts remain in how far the residual carious dentin might compromise the integrity of the restoration, as bond strengths to the remaining infected or affected dentin are reduced and softened dentin remains under the restoration. ${ }^{5-7}$ Moreover, it is criticised that the conventional assessment of selective carious tissue removal is arbitrary and unreliable, with subjective criteria like 'leathery' or 'reasonably soft' being used to describe what kind of dentin shall be left close to the pulp. ${ }^{8}$

Self-limiting polymer burs (like Polybur, Komet, Lemgo) abrade during carious tissue removal in sound dentin, which is harder than the bur. In contrast, they are supposed to remove carious dentin up to a certain hardness, afterwards they abrade once more, indicating the operator to stop carious tissue removal. ${ }^{9}$ Self-limiting burs thus standardise the conventional 'hardness' criterion used for carious tissue removal, which should increase reliability. These burs have been validated in vitro for selectivity (removing less sound dentin than conventional carious tissue removal) and have been further assessed for the bond strengths to the dentin walls remaining after their use. ${ }^{9-12}$

However, polymer burs have only sparsely been applied in clinical studies, most investigating peri-operative pain or the time needed when using such burs versus other instruments or criteria for carious tissue removal. ${ }^{8}$ It has so far not been shown that outcomes like pulp exposure or tooth survival are improved when using self-limiting burs ('objective removal') instead of conventional selective carious tissue removal to soft dentin ('subjective removal'). It also remains unknown how costly the use of both techniques is when considering initial costs (polymer burs are single-use products and more expensive than conventional steel or tungsten-carbide burs) and long-term costs (different risks of complications may lead to different needs for retreatment and, hence, costs). A systematic review ${ }^{8}$ therefore concluded that self-limiting polymer burs should be validated clinically against relevant outcomes, not only in vitro using surrogate parameters.

\section{Objectives}

Our study compares subjective with objective carious tissue removal of deep dentin lesions in primary molars with vital pulps. Our target population will be children from a high-needs community in Brazil, who will be followed over 3 years. We will compare the time until complications occur or the tooth needs removal and the subjective evaluation of both treatments. Our primary hypothesis is that the time until complications occur is significantly longer in teeth excavated using objective versus subjective criteria, that is, objective removal being superior. This assumption of superiority was made as self-limiting burs probably involve additional costs and efforts which need to be clinically justified.

\section{METHODS AND ANALYSIS}

\section{Overview}

The study is a community-based single-blinded clustered randomised controlled trial involving school children aged $6-8$ years. The present clinical trial is nested into a larger study that aims to evaluate the impact of oral health in children's development (quality of life, anthropometric and cognitive development). For this study, recruitment and treatment is provided at different public schools in Paranoá, a deprived suburban area of Brasilia, Brazil.

For our trial, recruitment has been finalised and patients are re-examined for the first recall visit (12 months) in 2018. We enrolled patients with one or more deeply carious, vital and non-symptomatic primary molar(s).

The unit of randomisation was the child, with all primary molars with a deep carious lesion in the same child receiving one of the two treatments. Non-eligible teeth in eligible patients were treated according to their need using standard protocols either on site or via referral to the paedodontic service of the University of Brasília. These protocols included atraumatic restorative treatment using a high-viscosity glass-ionomer cement or bulk-fill resin composite placed using a universal adhesive system.

Total follow-up time will be 3 years after completion of the initial treatment (ie, until 2020); longer follow-ups are not planned as children will change schools and teeth will exfoliate. Re-examinations will be performed after 12, 24 and 36 months. The study is registered at ClinicalTrials.gov (NCT02754466).

\section{Setting and participants}

Study recruitment took place in public primary schools of Paranoá, in Brasilia, the capital of Brazil. Participants were invited to attend routine examinations and recruited to the trial if eligible. Treatment was provided in a mobile dental unit, as described below. We included 115 children aged 6-8 years with minimum one vital, clinically and radiographically non-symptomatic, retainable, deeply carious primary molar. The lesion needed to radiographically extend into the inner half of the dentin and show signs of activity, for example, softness and roughness of the surface, plaque stagnation. Single and multiple surface lesions were included. The size of the cavity after preparation (maximum orovestibular extension and included surfaces) was recorded for later analysis of possible influences on outcomes. The lesion should not have been associated with non-carious hard tissue defects like fluorosis or molar incisor hypomineralisation. Patients required parental consent for participation. In addition, patients' cooperation for treatment under no or only local anaesthesia was needed. Patients with severe systemic diseases or disabilities were not included. Patients with known allergies to dental materials used within the study as well as those with teeth expected to exfoliate within the next 18 months were also not included. 


\section{Sample size}

The unit of analysis was the tooth. Hence, this a multilayered cluster randomised trial, with the patient and the school being the clusters. Clustering needs to be taken into account. This is done using the so-called Lee, Wei and Amato (LWA) model for clustered survival data. ${ }^{13}$ Sample size estimation for this model was based on the ideas of Xie and Waksman. ${ }^{14}$ The necessary calculations were performed with the $\mathrm{R}$ program ssizecl.surv. ${ }^{15}$ Required sample size was calculated based on the primary outcome parameter 'time until complications'. We anticipated that at 36 months, $90 \%$ of the objectively excavated teeth (polymer bur carious tissue removal) and $80 \%$ of the subjectively excavated teeth (subjective carious tissue removal) would not experience any complications; this is in the range of what has been reported for similar treatments. ${ }^{17}$ As mentioned, the assumption of superiority was made, as polymer burs involve additional costs and efforts, which need to be clinically justified. If $\alpha=0.05$ (two-sided) and $1-\beta=0.9$, a sample size of 45 children per group was needed when conservatively assuming an intercluster correlation (ICC) of 0.8 and a mean of 1.5 teeth per child being treated. Note that such ICC is relatively high, but accounts for not knowing the ICC in the specific population. Assuming a smaller ICC of 0.3 required 37 patients per group. Assuming an overall drop-out of $20 \%$, the required sample size was 57 per group. We eventually recruited 115 patients (177 teeth). Note that we had originally aimed to recruit an even higher number of children, but that this was not feasible within the financial scope of this trial.

\section{Recruitment}

Recruitment was performed within six primary schools in Brasília. A routine examination was offered to all potentially eligible pupils. Eligible patients and their parents received the study information and consent forms. Written consent was given by the parents of the children. There was a minimum time period of 48 hours between study information and consent.

\section{Allocation and blinding}

Random allocation was performed as follows. When the child was seated in the chair, one opaque envelope, containing the allocation, was drawn from a total of 120 envelopes (60 per group). Opening the envelope revealed the allocation. Note that eventually, only 115 children were recruited (as described), which is why groups are not perfectly balanced (five envelopes were never opened).

Blinding of the operator and the patient was not possible. However, clinical follow-up examinations will be performed blinded. This will be possible, as provided restorations will be identical in both groups, making it impossible to identify groups without further knowledge. We will also ensure that neither the child nor the parents will provide any information regarding the group allocation to the examiner. If patients require radiographic evaluation during follow-up visits, an independent third researcher will modify the radiographs in such way that identification of groups (eg, via assessing the thickness of remaining residual carious dentin) is not possible for the examiner. Note that not all patients may require follow-up radiographs and that further radiographs are usually only taken for patients requiring retreatments due to radiation protection reasons.

\section{Outcomes}

Outcomes will be assessed by an independent dentist who will be blinded for the original intervention. The primary outcome of the study will be the time until complications (endodontic and/or restorative) occur. Secondary outcomes will include the time until tooth removal is needed, patients' satisfaction with treatment using a 5-point Likert scale and cost-effectiveness (see below).

\section{Intervention and data collection}

Examination, intervention and data collection were or will be provided in a mobile dental unit. In the first examination visit, a full assessment and intraoral examination was performed. For patients who were possibly eligible, caries risk was estimated using the decayed missing filled teeth index (DMFT, measured using WHO criteria), with DMFT $\geq 2$ counting as high risk. If informed consent was given, treatment was provided in the second visit, with local anaesthetics being applied if needed (only two cases eventually required this; the decision if the discomfort was bearable was made by the child). Note that all patients had been conditioned for dental therapy a priori.

Removal of enamel and cavity preparation were performed using water-cooled diamond instruments; removal of peripheral carious dentin was performed using conventional rose head burs and/or hand excavators until only hard, dry dentin remains. Pulpo-axial carious dentin was removed either using a hand excavator until leathery, slightly moist and reasonably soft dentin remains (ie, dentin which cannot be removed using an excavator without force being needed; subjective removal) or via polymer burs (Polybur, Komet) until a new bur abrades and does not remove any dentin anymore (objective removal).

Operating dentists were calibrated prior to study commencement regarding these criteria and methods using extracted teeth. Moisture control was performed using cotton rolls and continuous aspiration. Restorations were performed using a high-viscosity glass ionomer cement (Equia Forte, GC, Tokyo, Japan).

Pulpal exposure did not occur. Follow-up treatment will be provided according to standardised local dental routine. All retained teeth will be followed regardless of the treatment arm.

Data collection will be performed via case reports forms. The following data have been or will be collected:

a) At the initial examination:

- Age, gender, medical and dental history;

- Caries risk; 
Table 1 Timing of measurements

\begin{tabular}{|c|c|c|c|c|}
\hline Measures & Preoperative & $\begin{array}{l}\text { Carious } \\
\text { tissue } \\
\text { removal and } \\
\text { restoration }\end{array}$ & $\begin{array}{l}\text { Follow- } \\
\text { up }\end{array}$ & Retreatment \\
\hline Demographics & $x$ & & & \\
\hline Dental history & $x$ & & & \\
\hline Caries risk & $x$ & & & \\
\hline $\begin{array}{l}\text { Caries } \\
\text { Assessment } \\
\text { Spectrum and } \\
\text { Treatment score }\end{array}$ & $x$ & & & \\
\hline $\begin{array}{l}\text { Radiographic } \\
\text { depth }\end{array}$ & $x$ & & & \\
\hline $\begin{array}{l}\text { Subjective } \\
\text { assessment by } \\
\text { patients }\end{array}$ & & $x$ & & $x$ \\
\hline $\begin{array}{l}\text { Costs (time, staff, } \\
\text { material) }\end{array}$ & & $x$ & & $x$ \\
\hline Clinical outcomes & & & $x$ & $x$ \\
\hline
\end{tabular}

- Decayed, missing, filled teeth according to WHO criteria;

- For teeth which are potentially eligible: Caries Assessment Spectrum and Treatment code, ${ }^{16}$ involved surfaces and cavity size, extension, lesion activity, radiographic lesion depth;
- Endodontic status: absence of spontaneous pain, no swelling or sinus formation.

b) After treatment:

- Operator;

- Surfaces treated;

- Used anaesthetic material;

- Subjective assessment (satisfaction) after the treatment (a 5-point Likert face scale was used), and the question 'how satisfied were you with this treatment?' was asked;

- Transportation time and costs;

- Time, staff and material required for treatment.

c) At follow-up:

- Tooth retreated alieno loco (yes/no);

- Teeth extracted or extraction needed (yes/no);

- Tooth exfoliated (yes/no);

- Complications: pulpal complications (pain, pulpitis, sensitivity to percussion or cold/hot, swelling, sinus formation) non-pulpal complications (restorative need to re-intervene, eg, caries at restoration margins, fracture or loss the restoration; fracture of the tooth). d) After follow-up treatments:

- If follow-up treatments are required, costs of these will be assessed as well. Retained teeth will be followed-up regardless if they required retreatment or not to assess their survival. A summary of

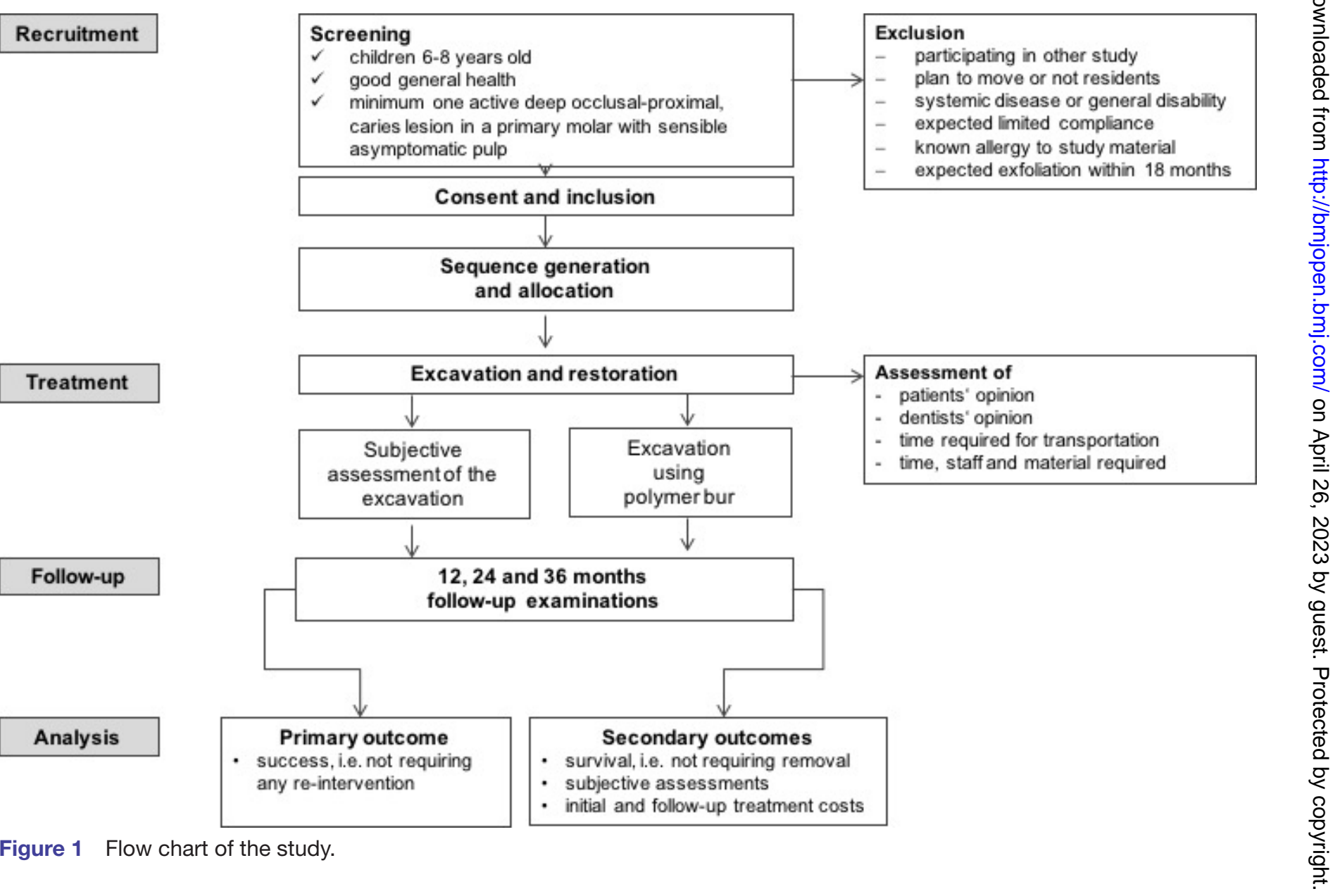


performed procedures and recorded data can be found in table 1.

The study flow is summarised in figure 1 .

\section{Data analysis and statistical evaluation}

As outlined the primary efficacy analysis will use a variant of the Cox proportional hazards model, that is, the LWA model that allows for multiple observations per subject to compare both treatment groups, at 36 months. This analysis will be performed with proc phreg (SAS V.9.4). Details may be found in the study by Guo. ${ }^{17}$ The primary analysis population will be based on the intention-to-treat principle (ITT). The ITT population is defined as the population who received at least one dental treatment (full analysis set); patients who have failed at time zero will be reported but will not provide data for the survival analysis. Additionally, a per-protocol analysis will be performed. A sensitivity analysis with regard to missing values will use multiple imputation methods. ${ }^{18}$ No interim analyses are planned. As this will be the single confirmatory analysis, no adjustment for multiple testing is required.

All secondary analyses will be exploratory. In this sense, we will analyse effects of possible confounders (school, age, gender, dental anxiety, caries risk, cavity size and number of involved surfaces, dental arch, first or second molar, surfaces). Mixed models will be used to compare subjective satisfaction and costs between groups. Incremental cost-effectiveness ratios and cost-effectiveness acceptability via the net-benefit approach will be used to describe the relative cost-effectiveness.

\section{Missing data}

We anticipate various reasons for missing data (drop-out, complications, withdrawal, adverse events). Sample size calculation was performed under the consideration of possible loss-to-follow-up, that is, data missing at random. To prevent data missing systematically, that is, not at random, case report forms were designed in a way enforcing complete reporting. We will account for data not missing at random according to the ITT principle in our primary analysis.

\section{Ethics and dissemination}

In case of adverse events (AEs) and/or severe adverse events (SAEs), the committee will be informed (see below). Both parents and patients will receive detailed verbal and written explanations regarding the study and the procedures therein involved. Informed written consent of the parents was required and was given not earlier than 48 hours after study information.

All amendments to the protocol shall be agreed to by the principal investigator (LAH) and be recorded with a justification for the amendments. Amendments will be reviewed to determine the need for ethically re-approving the amended protocol. Amendments will be recorded and reported appropriately.

Patients and their parents were informed that patients have the right to withdraw from the study at any time without giving reasons. Withdrawal will be documented and consent will be sought from participants to retain data collected up to the point of withdrawal.

We expect only few specific AEs or SAEs in our trial, like allergy or severe reaction to a study material used (local anaesthetics, restoration material) or rare, uncommon reactions (unexpected bleeding, severe pain, uncontrollable anxiety) to or during the performed interventions. Any $\mathrm{AE}$ will be investigated and reported to the ethics committee. In case of SAEs requiring unblinding, codebreaking is possible. If the investigation is terminated prematurely or suspended, the principal clinical investigator (LAH) will execute this termination and inform the ethics committee accordingly.

The results of this study will be published in international peer-reviewed journals. A summary of study results will also be saved at ClinicalTrials.gov to allow general access to obtained findings. Original data will be made available by authors on request.

\section{Patient and public involvement}

Neither patients nor the public were involved in the design of the study, including conceiving the research questions and deciding the outcomes. However, our study will assess the subjective burden on the patients as described.

\section{Trial status}

The trial was registered at ClinicalTrials.gov and the study recruitment has been finished.

\section{DISCUSSION}

The treatment of deep caries lesions in primary teeth is challenging for clinicians, which might be the reason why many carious primary teeth are not treated at all. ${ }^{19}$ While providing no treatment at all might be problematic in case of subsequent infection and pain, providing the conventional treatment via non-selective removal also may lead to loss of pulpal vitality and the need to re-intervene. ${ }^{20}$ Thus, selective removal, alongside sealing or non-restorative management approaches ${ }^{21}$ are increasingly suggested for treating such teeth, aiming at maintaining pulpal health and controlling the disease instead of removing its symptoms. ${ }^{22}$ However, the acceptance of these options among practitioners remains low, and the potential harm caused by remaining bacteria or the reduced longevity of the restorations placed onto carious dentin are cited as reasons for this resistance. ${ }^{23}{ }^{24}$ Using a removal method which determines when to stop removal could improve clinical outcomes and increasing the acceptance of selective carious tissue removal among practitioners. Moreover, it may allow standardising removal for scientific purposes and could thus make trials more comparable.

The planned study aims to compare selective removal using subjective criteria versus more objective polymer bur (self-limiting) excavation. We will evaluate three classes of outcome parameters: first, time until complications occur or removal. It should be noted that the clinical assessment 
is likely to underestimate the true risk of pulpal failures, as radiographic complications like inter-radicular lesions or resorptions might not be detected. However, this is true for most clinical situations, as young children will not be regularly followed up radiographically. It is also unclear what kind of treatment would be performed by dentists in case of an inter-radicular lesion, for example, being detected in a clinically asymptomatic primary molar in a child. ${ }^{25}$ Second, we will evaluate subjective outcomes, that is, those reported by patients. This is relevant, as especially for children, the subjective perception of dental treatment has huge impact on future attitudes towards dental visits and treatments: different levels of pain during treatment and different needs to invasively intervene can be supposed to impact on dental anxiety. Last, our study will evaluate costs and cost-effectiveness. This is relevant, as dental treatments are extremely costly, for example, the current EU27 spends $€ 79$ billion annually on dental care, predicted to reach $€ 93$ by $2020 .^{26}$ Demonstrating that certain strategies are clinically beneficial and accepted, and cost-effective, will be helpful for future decision makers. Moreover, costs will likely play a role in patients' decisions, especially when considering the distribution of deep lesions: the majority of lesions are found in few socioeconomically disadvantaged patients. ${ }^{27}$ Showing that treatments are cost-effective might thus also help to limit disincentives to demand dental services, especially among those who can barely afford them. ${ }^{28}$

There are certain limitations of the planned trial: first, we would have liked to use a defined core outcome set during the design of this trial. ${ }^{29}$ However, no such set is available at the moment; we thus attempted to define a wide set of outcomes as outlined. Second, this trial is conducted in a community setting in Brazil, which might limit its generalisability to other situations. Last and as discussed, our primary outcome might not be fully recording the true pulpal condition, but is clinically relevant for decision-making, which is why we accept this caveat.

Acknowledgements The authors would like to thank Komet, Lemgo, Germany, for kindly supporting this study with materials and covering material costs. The authors would like to thank GC Europe for providing the restoration material. The authors would also like to thank the Brazilian Federal District Research Support Foundation (FAPDF) for the provided research grant.

Contributors FS, SP, SL, APDR and LAH conceived the study and its design and participated in its coordination. FS and LAH drafted the protocol in accordance with international standards for clinical trials. PS performed the sample size calculation and supervised the planning of the statistical evaluation. MGM was responsible for children recruitment and treatments. All authors participated in the design of the study and read and approved the final protocol.

Funding The study will be supported by Komet, Lemgo, Germany, with polymer burs and funding for materials needed in the trial. GC Europe will provide the restoration material. The study received a research grant from the Brazilian Federal District Research Support Foundation (FAPDF, grant number 0193.001463/2016). No further funding was acquired.

Disclaimer The funders had no role in study.

Competing interests None declared.

Patient consent Not required.
Ethics approval School of Health Sciences of the University of Brasilia, protocol CAAE 51310415.0.0000.0030.

Provenance and peer review Not commissioned; externally peer reviewed.

Open access This is an open access article distributed in accordance with the Creative Commons Attribution Non Commercial (CC BY-NC 4.0) license, which permits others to distribute, remix, adapt, build upon this work non-commercially, and license their derivative works on different terms, provided the original work is properly cited, appropriate credit is given, any changes made indicated, and the use is non-commercial. See: http://creativecommons.org/licenses/by-nc/4.0/.

\section{REFERENCES}

1. Ricketts D, Lamont T, Innes NP, et al. Operative caries management in adults and children. Cochrane Database Syst Rev 2013;28:CD003808.

2. Schwendicke F, Stolpe M, Innes N. Conventional treatment, Hall Technique or immediate pulpotomy for carious primary molars: a cost-effectiveness analysis. Int Endod J 2016;49:817-26.

3. Oong EM, Griffin SO, Kohn WG, et al. The effect of dental sealants on bacteria levels in caries lesions. J Am Dent Assoc 2008;139:271-8.

4. Schwendicke F, Meyer-Lueckel H, Dörfer C, et al. Failure of incompletely excavated teeth--a systematic review. J Dent 2013;41:569-80.

5. Schwendicke F, Kern M, Meyer-Lueckel H, et alFracture resistance and cuspal deflection of incompletely excavated teeth. J Dent 2014;42:107-13.

6. Schwendicke F, Kern M, Blunck U, et al. Marginal integrity and secondary caries of selectively excavated teeth in vitro. $J$ Dent 2014;42:1261-8.

7. Hevinga MA, Opdam NJ, Frencken JE, et al. Does incomplete caries removal reduce strength of restored teeth? J Dent Res 2010;89:1270-5.

8. Schwendicke F, Paris S, Tu YK. Effects of using different criteria for caries removal: a systematic review and network meta-analysis. $J$ Dent 2015;43:1-15.

9. Boston DW. New device for selective dentin caries removal. Quintessence Int 2003;34:678-85.

10. Meller C, Welk A, Zeligowski T, et al. Comparison of dentin caries excavation with polymer and conventional tungsten carbide burs. Quintessence Int 2007;38:565-9.

11. Prabhakar A, Kiran NK. Clinical evaluation of polyamide polymer burs for selective carious dentin removal. J Contemp Dent Pract 2009;10:26-34.

12. Silva NR, Carvalho RM, Pegoraro LF, et al. Evaluation of a self-limiting concept in dentinal caries removal. J Dent Res 2006;85:282-6.

13. Lee $\mathrm{E}$, Wei LJ, Amato DA. Cox-type regression analysis for large number of small groups of correlated failure time observation. In: Klein JP, Goel PK, eds. Survival Analysis: State of The Art. Dordrect: Kluwer Academic, 1992:227-47.

14. Xie T, Waksman J. Design and sample size estimation in clinical trials with clustered survival times as the primary endpoint. Stat Med 2003;22:2835-46.

15. Campbell MJ, Walter SJ, Design H. Analyse and Report Cluster Randomised Trials in Medicine and Health Related Research. Chichester: Wiley, 2014.

16. de Souza AL, van der Sanden WJ, Leal SC, et al. The Caries Assessment Spectrum and Treatment (CAST) index: face and content validation. Int Dent J 2012;62:270-6.

17. Guo S. Survival Analysis. oxford: Oxford University Press, 2010.

18. Schafer JL. Multiple imputation: a primer. Stat Methods Med Res 1999;8:3-15.

19. Tickle M, Milsom K, King D, et al. The fate of the carious primary teeth of children who regularly attend the general dental service. $\mathrm{Br}$ Dent J 2002;192:219-23.

20. Innes NP, Evans DJ, Stirrups DR. The Hall Technique; a randomized controlled clinical trial of a novel method of managing carious primary molars in general dental practice: acceptability of the technique and outcomes at 23 months. BMC Oral Health 2007;7:18.

21. Santamaria RM, Innes NP, Machiulskiene V, et al. Acceptability of different caries management methods for primary molars in a RCT. Int J Paediatr Dent 2015;25:9-17.

22. Schwendicke F, Frencken JE, Bjørndal L, et al. Managing carious lesions: consensus recommendations on carious tissue removal. Adv Dent Res 2016;28:58-67.

23. Schwendicke F, Meyer-Lueckel H, Dörfer C, et al. Attitudes and behaviour regarding deep dentin caries removal: a survey among German dentists. Caries Res 2013;47:566-73. 
24. Schwendicke F, Stangvaltaite L, Holmgren C, et al. Dentists' attitudes and behaviour regarding deep carious lesion management: a multinational survey. Clin Oral Investig 2017;21:191-8.

25. Bowen JL, Mathu-Muju KR, Nash DA, et al. Pediatric and genera dentists' attitudes toward pulp therapy for primary teeth. Pediatr Dent 2012;34:210-5.

26. Petersen PE, Bourgeois D, Ogawa $\mathrm{H}$, et al. The global burden of oral diseases and risks to oral health. Bull World Health Organ 2005;83:661-9.
27. Ridell $\mathrm{K}$, Olsson $\mathrm{H}$, Mejàre I. Unrestored dentin caries and deep dentin restorations in Swedish adolescents. Caries Res 2008;42:164-70.

28. Schwendicke F, Paris S, Stolpe M. Cost-effectiveness of caries excavations in different risk groups - a micro-simulation study. BMC Oral Health 2014;14:153.

29. Lamont T, Schwendicke F, Innes N. Why we need a core outcome set for trials of interventions for prevention and management of caries. Evid Based Dent 2015;16:66-8. 